\title{
Combination Syndrome
}

\section{Sridharan Rajendran, Baburajan}

\section{ABSTRACT}

Combination syndrome (CS) is a dental condition that is commonly seen in patients with a completely edentulous maxilla and partially edentulous mandible with preserved anterior teeth. This syndrome consists of severe anterior maxillary resorption combined with hypertrophic and atrophic changes in different quadrants of maxilla and mandible. This makes it a challenging condition in dentistry that requires significant experience along with advanced restorative and surgical skills. The causes of maxillary bone resorption and ways to correct it are discussed in this article. Conventional treatment with full upper and partial lower dentures for the CS patients is not always adequate or satisfying for patients and it often requires multiple remakes due to continuing bone resorption. Dental implant rehabilitation challenges conventional treatment with bone-anchoring techniques to provide improved retention and stability for implant-retained and supported prostheses. This article presents a successful implant treatment of the partially edentulous maxilla in CS patient. The necessity of a multidisciplinary approach for early prevention and treatment of this complex condition is emphasized. This article illustrates a review of the changes and difficulties faced when treated with conventional approach which can be overcome with the aid of implant-supported prosthesis.

Keywords: Combination syndrome, Resorbed maxilla, Flabby tissue, Atrophic bone.

How to cite this article: Rajendran S, Baburajan. Combination Syndrome. Int J Prosthodont Restor Dent 2012;2(4):156-160.

Source of support: Nil

Conflict of interest: None

\section{INTRODUCTION}

The glossary of prosthodontic terms defines combination syndrome's characteristic features that occur when an edentulous maxilla is opposed by natural mandibular anterior teeth, including loss of bone from the anterior portion of the maxillary ridge, overgrowth of the tuberosities, papillary hyperplasia of the hard palate's mucosa, extrusion of the lower anterior teeth and loss of alveolar bone and ridge height beneath the mandibular removable dental prosthesis bases-also called anterior hyperfunction syndrome. ${ }^{1,2}$

There may be seven characteristics associated with this syndrome: (1) Bone loss in the premaxilla, (2) dropping of the posterior maxilla (tuberosities), (3) extrusion of the lower anterior teeth, (4) posterior bone loss in the mandible under the RPD, (5) papillary hyperplasia of the maxilla, (6) decreased occlusal vertical dimension and (7) facial esthetics often altered dramatically.

\section{Mechanics Which Produce the Combination Syndrome}

Negative pressure within the maxillary denture pulls the tuberosities down by which the anterior ridge is driven upward by the anterior occlusion thereby functional load will then direct stress to the mandibular distal extension resulting in bony resorption of the posterior mandibular ridge, supraeruption of mandibular anteriors, occlusal plane discrepancy, loss of vertical dimension of occlusion and chronic stress results in an ill-fitting prosthesis. ${ }^{3}$

Prevalence among denture patients: Among 150 patients who had completed maxillary dentures and mandibular anterior natural teeth, one in four demonstrated changes consistent with the diagnosis of combination syndrome (Shen and Gongloff 1989). ${ }^{4}$

\section{Classification of Combination Syndrome}

Kelly was the first person to use the term 'combination syndrome'. ${ }^{5}$ He believed that the key to many symptoms of the combination syndrome (CS) is the 'early loss of bone from the anterior part of the maxillary jaw'. ${ }^{3}$ The other consistent features of this dental condition include enlargement of maxillary tuberosities and mandibular posterior bone resorption that can be present in many but not all CS cases. Based on a literature review and the author's experience with a variety of combination syndrome patients (complete and partial maxillary and mandibular edentulous cases), a clinically relevant classification of CS is proposed. Three classes and 10 modifications of CS are described below. An anterior maxillary resorption resulting from the force of anterior mandibular teeth is the key feature of this classification, and it is consistently present throughout all classes and all modifications. 'Maxillary edentulous condition' defines the class, 'mandibular' the modification within the class. A treatment for each category of patients with CS is suggested:

- Class I: Maxilla: Completely edentulous alveolar ridge. Mandible: Modification 1 (M1): Partially edentulous ridge with preserved anterior teeth only. Modification 2 (M2): Stable 'fixed' full dentition (natural teeth or implant-supported crowns/bridges). Modification 3 (M3): Partially edentulous ridge with preserved teeth in anterior and one posterior region.

- Class II: Maxilla: Partially edentulous alveolar ridge with teeth present in both posterior regions, edentulous 
and atrophic anterior region. Mandible: Modifications are the same as in class I (M1, M2 and M3).

- Class III: Maxilla: Partially edentulous alveolar ridge with teeth present in one posterior region only, edentulous and atrophic anterior and one posterior region. Mandible: Modifications are consistent with class I and II (M1, M2, M3A and M3B) (Flow Charts 1 and 2). This classification is based on what seems to be the dominant feature of most CS cases-an edentulous premaxilla with an advanced resorption of anterior maxillary bone and overgrowth of the anterior mandibular bone with extrusion (supereruption) of lower front teeth.

\section{Treatment}

\section{Systemic and Dental Considerations}

- Review medical and dental history.

- Clinical and radiographic evaluation of both hard and soft tissues.

- Resolution of any inflammation, if present.

- Evaluation of patient's caries susceptibility, periodontal status and oral hygiene.

- Factors to be considered in tooth to be used as abutment. Kelly said that before proceeding with the prosthetic treatment, gross changes that have already taken place should be surgically treated. These include conditions like:

- Flabby (hyperplastic) tissue

- Papillary hyperplasia

- Enlarged tuberosities

- Lower partial denture base should be fully extended and should cover retromolar pad and buccal-shelf area.

\section{Treatment Planning}

When planning treatment for patients with edentulous maxillae and a partially edentulous mandible, the risk of development of the CS must be recognized.

\section{Basic Treatment Objective}

Develop an occlusal scheme that discourages excessive occlusal pressure on the maxillary anterior region, in both centric and eccentric positions (Saunders et al, 1979).

Treatment planning for the completely edentulous maxillary arch: Treatment modality should be using the principle of restoring a stable posterior occlusion, while minimizing occlusal pressures on the anterior maxilla. Prevention of the combination syndrome must be our primary objective. Planned extractions followed by immediate dentures or preservation of a few of the remaining teeth for the fabrication of overdenture prosthesis with a metallic denture base for one arch are preferred treatment modalities. This decreases the risk for occurrence of CS by preventing a completely edentulous arch from opposing natural teeth. For those patients in which CS has already occurred, conventional prosthodontic techniques with special consideration for flabby tissues must be followed or else a multidisciplinary approach may be followed. Surgical intervention (vestibuloplasty and excision of flabby tissue) followed by a metallic denture base prosthesis is the treatment of choice. Different patients with particular clinical findings should be treated specifically to prosthodontically rehabilitate them and prevent combination syndrome.

Flow Chart 1: Classification of CS

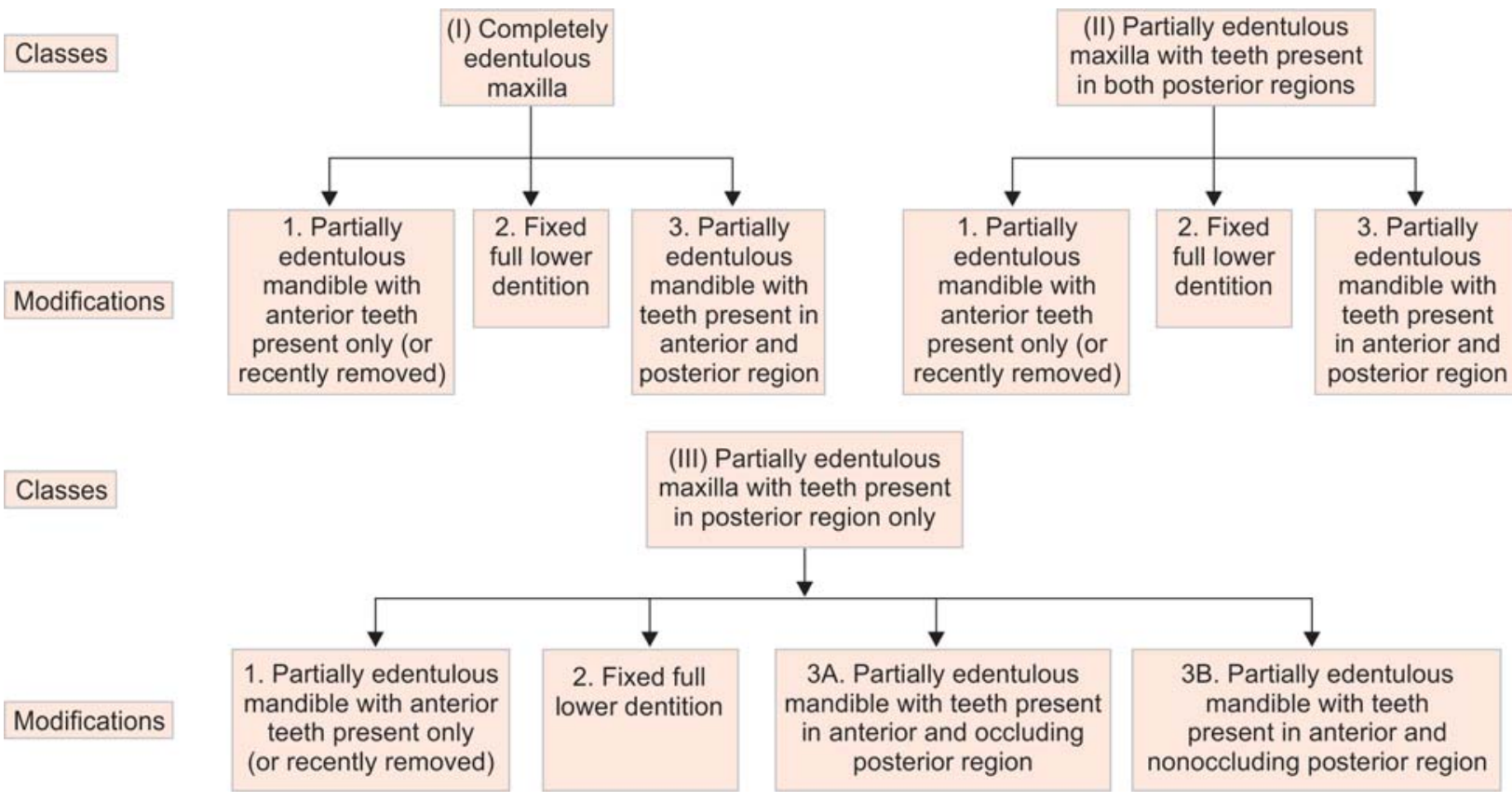


Flow Chart 2: Modifications of classes I and II

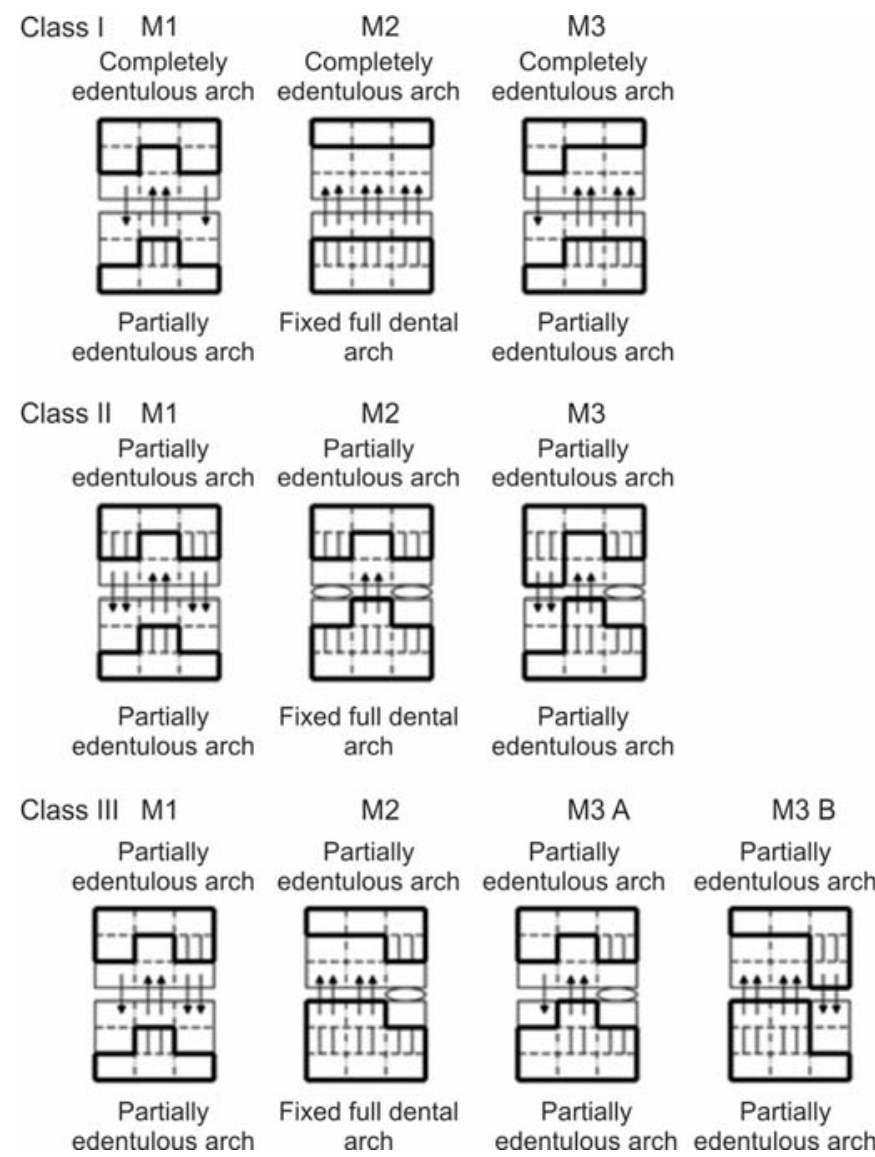

Treatment option 1: Planned extractions followed by immediate dentures-this treatment option is considered when arch relationship negates an overdenture and requires an alveolectomy along with extraction of the anterior teeth for patients reporting with severe prognathic maxilla, periodontally compromised proclined anterior teeth present in the maxillary arch and missing mandibular posterior teeth. The extractions of the maxillary anterior teeth were planned along with the alveolectomy in the maxillary anterior region. Hence, conventional immediate denture fabrication steps were followed during primary and secondary impression making, jaw relation recording, posterior try-in and mock.

Treatment option 2: Overdenture prosthesis with a metallic denture base-every effort should be made to avoid the potentially destructive occlusal forces exerted on the anterior maxillary residual ridge. Therefore, when a maxillary complete denture is contemplated, endodontic and periodontic techniques are used to preserve roots in order to maintain the bony architecture of the anterior maxilla.

Treatment option 3: Conventional prosthodontic techniques with special consideration for flabby tissues - a variety of techniques have been suggested to circumvent the difficulties of making a denture rest on flabby ridge. It has been stated that while the flabby ridge may provide poor retention for a denture, it is better than no ridge, as could occur following surgical excision of the flabby tissues.

Treatment option 4: Surgical intervention (vestibuloplasty and excision of flabby tissue) followed by metallic denture base prosthesis-patients reporting with a completely edentulous maxillary arch opposing anterior natural dentition in the mandibular arch along with destructive changes in the hard and soft tissues of the jaws of the combination syndrome, such as severe anterior ridge resorption, epulis fissuratum and flabby tissue in the maxillary arch accompanied by loss of vertical dimension require surgical intervention.

\section{Treatment Planning for the Distal Extension Partially Edentulous Mandibular Arch}

The main aim of prosthetic therapy is to restore stable occlusion with good facial height. The main risk factors to consider in the partial edentulous patients are associated with the progression of periodontal disease, caries activity, residual ridge resorption and functional problems. It is often possible to stop progression of periodontal disease in the elderly with conservative and surgical periodontal therapy; and by instituting adequate hygiene measures. In this way stable periodontal conditions can normally be established, which are essential for an acceptable long-term prognosis of reconstructive prosthodontic therapy. Different treatment approaches have been advocated for the low-risk patients who have not yet developed the combination syndrome and with well-preserved mandibular anterior teeth and the highrisk patients with destructive changes or supraerupted or periodontally involved mandibular anterior teeth. The spatial position of the mandibular anterior teeth is important to the treatment plan.

Treatment option 1: Overdenture-teeth that are considerably supraerupted would require alteration by shortening, crowning or placing them under an overdenture to obtain a harmonious occlusion. The spatial position of the mandibular anterior teeth is important to the treatment plan. The level of the incisal edges of the mandibular anterior teeth should be assessed in comparison to the proposed posterior occlusal plane (Fig. 1).

Treatment option 2: A removable cast partial dentureMouth preparation is done to support a removable cast partial denture with an occlusal plane conducive to a bilateral balanced articulation. The lingual plate delays the overeruption of the mandibular teeth, preventing undesirable anterior pressure on the anterior part of the maxillary denture. Optimum fit of the denture base of the removable cast partial denture is achieved using the altered cast technique. Posterior occlusal contact must be maintained 

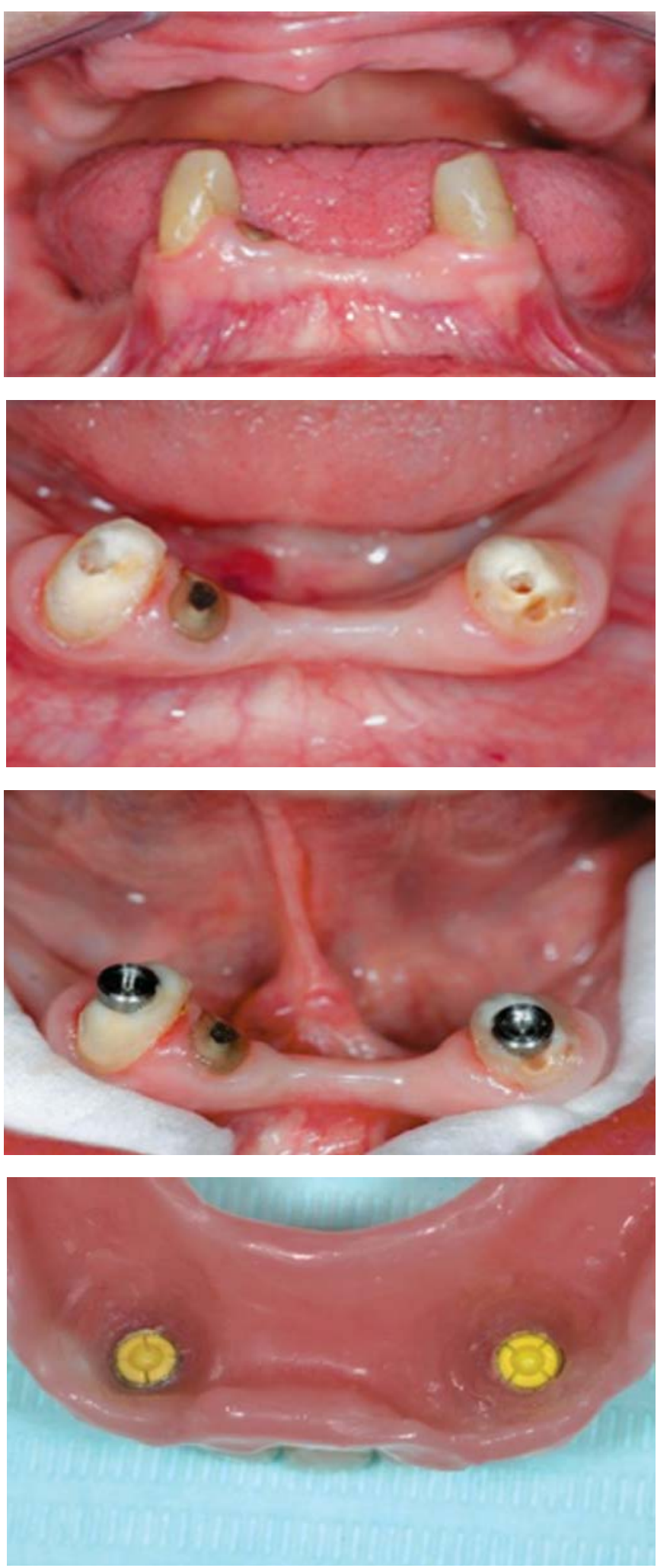

Fig. 1: Lower overdenture with intracoronal retainers

by constant relining of the distal extension denture base to compensate for its resorption (Fig. 2).

Treatment option 3: Implant-supported fixed prosthesisin distal extension partially edentulous situations implantsupported fixed prosthesis may be used in case there is adequate bone height and width, no anatomic structures that
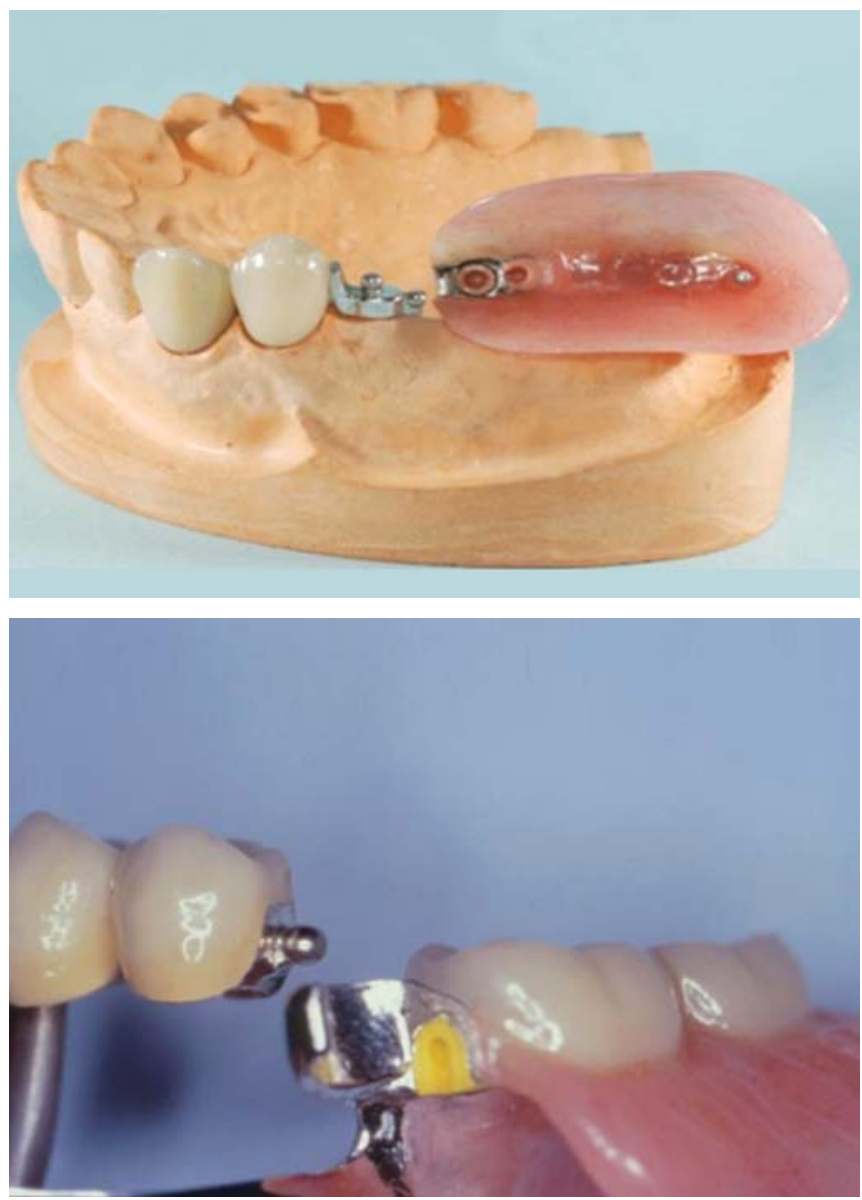

Fig. 2: Lower precision attachments

could interfere with implant placement and visual inspection and palpation do not show presence of any flabby excess tissue, bony ridges and sharp underlying osseous formations or undercuts. Either an implant can be placed distal to the most posterior natural abutment and a fixed prosthesis connecting the implant with the natural teeth can be fabricated or two or more implants can be placed posterior to the most distal natural tooth in order to fabricate a completely implant-supported restoration (Fig. 3).

\section{DISCUSSION}

Different treatment approaches have been suggested for patients with an edentulous maxilla and some remaining anterior mandibular teeth. The choice of treatment ultimately depends on the patient, the amount of time and money he is willing to spend for the treatment, his oral condition and his desire for fixed or removable prosthesis. Some patients demand implants and some refuse them. Advantages of implants compared to the conventional prosthodontics are that implants stimulate the bone and maintain its dimension similar to natural teeth. ${ }^{6}$ Maxson et $\mathrm{al}^{7}$ report similarities between patients with overdentures constructed on transmandibular implants that oppose complete maxillary dentures, hence, only endosseous implants must be used. 


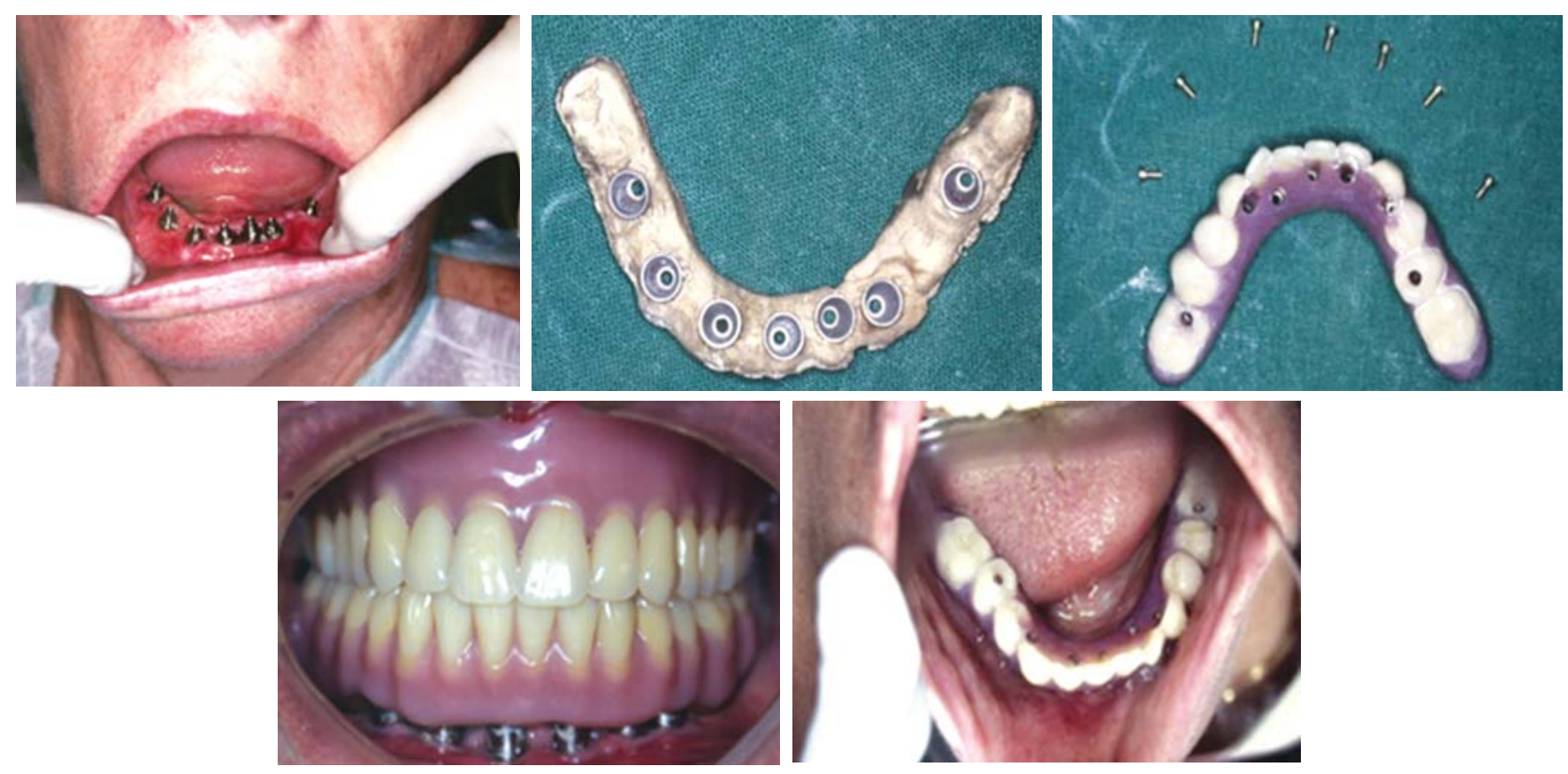

Fig. 3: Lower implant-supported complete denture

Implants provide a predictable method of tooth replacement offering excellent functional and esthetic benefits. Following the same prosthetic concepts for the maxilla as existed in the mandible is not feasible. The long-term prognosis for implants in the maxilla is less secure than that of the edentulous mandible. Following tooth extraction in the anterior part of the maxilla, horizontal bone resorption is almost twice as pronounced as vertical resorption. The reduced quantity and quality of bone in the maxilla together with increased esthetic demands makes treatment planning more complex. A different approach based on the ultimate esthetic outcome is required compared to that of the edentulous mandible, where function is the more critical factor.

\section{CONCLUSION}

The evaluation of the risk of developing the combination syndrome is based on dental history and the condition of the remaining mandibular anterior teeth. High-risk patients showing changes associated with the syndrome are more likely to be those who stress the maxillary ridge, such as in Angle class III jaw relationships, parafunctional habits and in patients who have functioned mainly with mandibular anterior teeth for long periods. The degenerative changes that develop in the edentulous regions of wearers of complete upper and partial lower dentures are almost inevitable. The dentist must carefully plan the treatment of these patients in order to maintain the health of the oral tissues of these patients and provide them with prosthesis that provide function but do not contribute to the combination syndrome. Thorough diagnosis, planning and implementation of treatment will result in an outstanding outcome for both the patient and dentist.

\section{REFERENCES}

1. Kelly E. Changes caused by a mandibular removable partial denture opposing a maxillary complete denture. J Prosthet Dent 1972;27(2):140-50.

2. Palmqvist $\mathrm{S}$. The combination syndrome: A literature review-J Prosthet Dent 2003;90(3),270-75.

3. Saunders T, Gillis Jr R, Desjardins R. The maxillary denture opposing the mandibular bilaterall distal extension partial denture. Treatment options. J Prosthet Dent 1979;41(2):124-28.

4. Koper A. The maxillary complete denture opposing natural teeth -problems and solutions. J Prosthet Dent 19878;57(6);704-07.

5. Schmitt S. Combination syndrome-a treatment approach. J Prosthet Dent 1985;54(5):664-71.

6. Chee W, Jivraj S. Treatment planning of the edentulous mandible. British Dent J 2006;201(6):337-48.

7. Maxson B, Powers M, Scott R. Prosthodontic considerations for the transmandibular implant. J Prosthet Dent 1990;63(5): 554-58.

\section{ABOUT THE AUTHORS}

\section{Sridharan Rajendran}

Professor, Department of Prosthodontics, Chettinad Dental College Chennai, Tamil Nadu, India

\section{Baburajan (Corresponding Author)}

Professor, Department of Prosthodontics, Mahatma Gandhi Dental College, Puducherry, India, e-mail: drbaburajan@gmail.com 